\title{
COVID-19 Vaccination and Timing of Bariatric Surgery: Considerations and Concerns for Patients with Severe Obesity
}

\author{
Abdolreza Pazouki ${ }^{1,2,3} \cdot$ Shahab Shahabi ${ }^{1,2,3}$ - Erfan Sheikhbahaei ${ }^{4} \cdot$ Sommayeh Mokhber $^{1,2,3}$. \\ Amir Hossein Davarpanah Jazi ${ }^{1,2,3} \cdot$ Mohammad Kermansaravi $^{1,2,3}$
}

Received: 22 August 2021 / Revised: 22 September 2021 / Accepted: 22 September 2021 / Published online: 28 September 2021

(c) The Author(s), under exclusive licence to Springer Science+Business Media, LLC, part of Springer Nature 2021

\section{Dear Editor,}

In COVID-19 pandemic, vaccination plays a crucial role in prevention, decreasing the symptoms and COVID-related mortality [1,2], especially in high-risk populations such as patients with severe obesity [3-6]. Different COVID-19 vaccines have been developed and administered worldwide and the size of the vaccinated population is growing; however, the speed of vaccination, anti-vaccine movements, the emergence of new SARS-CoV-2 variants (i.e., Delta and Lambda strains), and the failures to adhere to the safety protocols continue to extend the pandemic and create further challenges $[1,7]$.

As shown in literature, obesity is itself an independent risk factor for COVID-19, and obese patients may suffer

Shahab Shahabi

shshahabi@yahoo.com

Abdolreza Pazouki

apazouki@yahoo.com

Erfan Sheikhbahaei

e.shikhbahaei@edc.mui.ac.ir

Sommayeh Mokhber

dr_so_mokhber@yahoo.com

Amir Hossein Davarpanah Jazi

davarpanahjazi@gmail.com

Mohammad Kermansaravi

mkermansaravi@yahoo.com

1 Minimally Invasive Surgery Research Center, Hazrat-E Rasool General Hospital, Iran University of Medical Sciences, Masouri St. Niyayesh St. Satarkhan Ave., Tehran, Iran

2 Iran National Center of Excellence for Minimally Invasive Surgery Education, Iran University of Medical Sciences, Tehran, Iran

3 Center of Excellence of European Branch of International Federation for Surgery of Obesity, Tehran, Iran

4 Isfahan Minimally Invasive Surgery and Obesity Research Center, Isfahan University of Medical Sciences, Isfahan, Iran more severe COVID-19 symptoms [8, 9]. Although elective bariatric procedures can be performed when the safety protocols are fully observed, there are certain measures to consider. First, elective surgeries should not be pursued in health centers admitting COVID-19 patients; second, PCR for all candidates as well as CT scanning for symptomatic patients are advised before surgery; third, patients should be discharged from the hospital as quickly as possible after their operation [7]. The paradox, however, lies in that some bariatric surgery candidates might not have received COVID-19 vaccines before their surgery out of choice in some countries, while in others, access to the COVID-19 vaccines is scarce; this situation makes decision-making about elective surgeries even more challenging [10]. The public trust in COVID-19 vaccines is a little disappointing around the world [10-12]; however, it is not feasible to force patients to get vaccinated before their surgery either. Although vaccination is preferred from a medical perspective, not all patients can be expected to be fully vaccinated, especially those in countries that are behind on their vaccine schedules or lack sufficient vaccine supplies. If the patient does get vaccinated, the minimum required interval between bariatric surgery and the first shot of the vaccine is two weeks. This timing is recommended because vaccines may mask early postoperative complications, and bariatric surgery patients should be able to live and work on their own and start taking oral nutrients after their surgery. A shorter interval may unnecessarily increase the risk of surgical site infection or anastomosis leak and may also increase the risk of thromboembolism if the patient is not ambulated well or fully hydrated [3-6, 8]. In such scenarios, our preference is to operate in clean centers, screen for COVID infection (mostly through PCR testing), make sure the patient does not have any symptoms and has not had contact with infected patients, and encourage the patient to get vaccinated regardless of the vaccine brand.

The current recommendation is that surgery can be safely performed two weeks after the second shot of the 
COVID-19 vaccine [13], but we need to find out whether or not this reference also works for bariatric surgery [3-5]. Most vaccines may cause nonspecific symptoms, like a lowgrade fever, myalgia, headache, fatigue, and malaise, which mimic early postoperative complications such as anastomosis leaks, infections, and atelectasis, or they may enhance adverse anesthesia reactions. The recommendation is to take sufficient amounts of water after vaccination; however, the permitted oral intake decreases dramatically with any type of bariatric procedure, which may cause some degree of dehydration if the patient needs more hydration due to another condition or infection. In rare cases, some vaccines unpredictably cause thromboembolism [14]. Bariatric candidates are more prone to thromboembolic events due to their high $\mathrm{BMI}, \mathrm{CO}_{2}$ insufflation during operation, and steep anti-Trendelenburg position during the surgery along with their low physical activity and venous stasis, which increase the risk of thromboembolism if there is an underlying condition such as oral contraceptive use. Furthermore, certain bariatric procedures such as sleeve gastrectomy impose a higher risk of portomesenteric thrombosis [15]. Therefore, the timing between the last shot of the vaccine and bariatric surgery needs to be effectively addressed for the safety of our patients. Consequently, we do not recommend less than two-week interval between the second shot of the COVID-19 vaccine and bariatric surgery. This interval seems safe but needs more investigation in this specific group of patients. All vaccinated persons should know that full vaccination does not guarantee immunity from COVID-19, and as surgeons, we should adhere to the safety protocols just as our patients do. The reason is that if bariatric patients contract COVID-19 during the postoperative convalescent period, the risk of thromboembolic events and hospitalization becomes significant due to their low oral intake and limited physical activity $[8,9,12,16]$.

Another concern is about virus reactivation or actual COVID-19 infection after vaccination if the patients consume multivitamins. Multivitamins are widely prescribed in various forms after nearly all bariatric procedures. Literature does not support the hypothesis that live attenuated vaccines can cause COVID-19 solely as a result of multivitamin consumption [17, 18]. Therefore, if the patient does not show evidence of immunodeficiency disorders (e.g., has not been under chemotherapy or taken corticosteroids or other immunomodulating agents), there is no compelling evidence against the use of multivitamins after bariatric surgery. Nonetheless, a two-week interval between vaccine and surgery seems safe, and it is unlikely for any adverse immune reactions to occur after this two-week window.

Despite the lack of thorough investigation on this subject, obesity has been proposed to interact with immune function $[3,19]$, and it is therefore likely that weight loss by any intervention may enhance the immune system function $[3,20]$.
Nevertheless, we should note that any kind of surgery may put the patient in greater danger of immune system malfunction due to the surgery itself and other unknown mechanisms that occur in the body after surgery. Bariatric procedures facilitate substantial and sustainable weight loss, and some of them create a malabsorption state in the patients [21]. Although bariatric surgery is generally beneficial for people with severe obesity and obesity-related health conditions, we do not yet know how this surgery affects the immune system and immune cell functions.

Finally, we encourage operating on fully vaccinated patients. As for the non-vaccinated candidates, we recommend a two-week interval between the surgery and the first shot of the vaccine post-operation, or a two-week interval between the second shot of the vaccine and the surgery, with more preference for the latter due to its lower likelihood of infection after surgery. There is no problem with taking multivitamins after bariatric surgery. We highly recommend our patients ambulate as soon as possible and have sufficient oral intake both before vaccination after surgery or after vaccination before surgery.

Data Availability Not applicable.

Code Availability Not applicable.

\section{Declarations}

Ethics Approval N/A.

Consent to Participate N/A.

Consent for Publication Not applicable.

Conflict of Interest The authors declare no competing interests.

\section{References}

1. Paltiel AD, Schwartz JL, Zheng A, et al. Clinical outcomes of a COVID-19 vaccine: implementation over efficacy. Health Aff. 2021;40:42-52.

2. Shirani K, Sheikhbahaei E, Torkpour Z, et al. A narrative review of COVID-19: the new pandemic disease. Iran J Med Sci. 2020;45:233-49.

3. Kipshidze NNNN, Kipshidze NNNN, Fried M. COVID-19 vaccines: special considerations for the obese population. Obes Surg Obesity Surgery. 2021;31:3854-6.

4. Butsch WS, Hajduk A, Cardel MI, et al. COVID-19 vaccines are effective in people with obesity: a position statement from the obesity society. Obesity. 2021;29(10):1575-9.

5. Ryan DH, Ravussin E, Heymsfield S. COVID 19 and the patient with obesity - the editors speak out. Obesity. 2020;28:847.

6. Cuschieri S, Grech S. Obesity population at risk of COVID-19 complications. Glob Health Epidemiol Genom. 2020;5:e6. 
7. Wu K, Smith C, Lembcke B, et al. Elective surgery during the COVID-19 pandemic This. N Engl J Med. 2020;383:1787-90.

8. Marchesi F, Valente M, Riccò M, et al. Effects of bariatric surgery on COVID-19: a multicentric study from a high incidence area. Obes Surg Obesity Surgery. 2021;31:2477-88.

9. Singhal R, Ludwig C, Rudge G, et al. 30-Day morbidity and mortality of bariatric surgery during the COVID-19 pandemic: a multinational cohort study of 7704 patients from 42 countries. Obes Surg. 2021;30:1-7.

10. Lewis JR. What is driving the decline in people's willingness to take the COVID-19 vaccine in the United States? JAMA Heal Forum. 2020;1:e201393.

11. Khubchandani J, Sharma S, Price JH, et al. COVID-19 vaccination hesitancy in the United States: a rapid national assessment. J Community Health [Internet] Springer US. 2021;46:270-7. https://doi. org/10.1007/s10900-020-00958-x

12. Vallis $\mathrm{M}$, Glazer S. Protecting individuals living with overweight and obesity: attitudes and concerns toward COVID-19 vaccination in Canada. Obesity. 2021;29:1128-37.

13. El-Boghdadly K, Cook TM, Goodacre T, et al. SARS-CoV-2 infection, COVID-19 and timing of elective surgery: a multidisciplinary consensus statement on behalf of the Association of Anaesthetists, the Centre for Peri-operative Care, the Federation of Surgical Specialty Associations, the Royal College of Anaesthetists and the Royal College of Surgeons of England. Anaesthesia. 2021;76:940-6.

14. Østergaard SD, Schmidt M, Horváth-Puhó E, et al. Thromboembolism and the Oxford-AstraZeneca COVID-19 vaccine: side effect or coincidence? Lancet. 2021;397:1441-3.

15. Shaheen O, Siejka J, Thatigotla B, et al. A systematic review of portomesenteric vein thrombosis after sleeve gastrectomy. Surg
Obes Relat Dis [Internet] Elsevier. 2017;13:1422-31. https://doi. org/10.1016/j.soard.2017.03.015.

16. Singhal R, Tahrani AA, Ludwig C, et al. Global 30-day outcomes after bariatric surgery during the COVID-19 pandemic (GENEVA): an international cohort study. Lancet Diabetes Endocrinol. 2021;9:7-9.

17. Rayman MP, Calder PC. Optimising COVID-19 vaccine efficacy by ensuring nutritional adequacy. Br J Nutr. 2021;50:2-3.

18. Kumar P, Kumar M, Bedi O, et al. Role of vitamins and minerals as immunity boosters in COVID-19. Inflammopharmacology [Internet] Springer International Publishing. 2021;29:1001-16. https://doi.org/10.1007/s10787-021-00826-7.

19. Pellini R, Venuti A, Pimpinelli F, et al. Obesity may hamper SARS-CoV-2 vaccine immunogenicity strategies for COVID19, particularly in obese people. Funding: None Key words: COVID-19; SARS-CoV-2; vaccine; obesity; antibodies; serum titre. medRxiv [Internet]. 2021. 1-10. Available from: https:// www.medrxiv.org/content/10.1101/2021.02.24.21251664v1

20. Rao SR. Inflammatory markers and bariatric surgery: a metaanalysis. Inflamm Res. 2012;61:789-807.

21. Keleidari B, Mahmoudieh M, Shahabi S, et al. Reversing oneanastomosis gastric bypass surgery due to severe and refractory hypoalbuminemia. World J Surg. 2020;44(4):1200-8.

Publisher's Note Springer Nature remains neutral with regard to jurisdictional claims in published maps and institutional affiliations. 